\title{
ansa-Metallocene derivatives
}

\section{XVIII * Chiral titanocene derivatives accessible from substituted dihydropentalene and azulene precursors}

\author{
Peter Burger, Hans-Ulrich Hund, Kaspar Evertz and Hans-Herbert Brintzinger ** \\ Fakultät für Chemie, Universität Konstanz, D-7750 Konstanz (F.R.G.)
}

\begin{abstract}
The product of an $\mathrm{Mg} / \mathrm{CCl}_{4}$-induced reductive coupling of guaiazulene reacts with $\mathrm{TiCl}_{3} \cdot 3 \mathrm{THF}$ to yield, after oxidation with $\mathrm{HCl} / \mathrm{O}_{2}, R, S-8,8^{\prime}$-biguaiazulenide titanium dichloride, which contains a $\mathrm{C}-\mathrm{C}$ bond between its two guaiazulenide ligand moieties. A similar reaction sequence starting from 3-phenyl-1,2-dihydropentalene gives unbridged $R, S$-bis(4-exo-phenyl-4H-5,6-dihydropentalenide)titanium dichloride.
\end{abstract}

\section{Introduction}

Chiral ferrocene derivatives which contain $\mathrm{C}-\mathrm{C}$ coupled biazulenide or bipentalenide *** ligand frameworks were prepared some years ago by Fischer and Müller [2] and by Katz and coworkers [3], and were structurally characterized by Churchill and coworkers [4,5]. We recently observed that substituted pentafulvenes can be reductively coupled with magnesium metal and $\mathrm{CCl}_{4}$ to ethanediyl-bridged bis(cyclopentadienide- $\mathrm{MgCl}$ ) derivatives [6], which are particularly well suited for the synthesis of chiral ansa-metallocenes by direct metathetical reaction with the appropriate transition metal halide. We have now examined the possibility of using such coupling to obtain related, chiral titanocene derivatives containing two bicyclic ligand framework moieties bridged by a $\mathrm{C}-\mathrm{C}$ bond.

\footnotetext{
* For part XVII see ref. 1.

** To whom correspondence should be addressed.

*** This nomenclature is proposed for the dianions of the respective hydrocarbon dimers and their metal derivatives, in distinction to "bis" complexes containing two unconnected ligand moieties.
} 

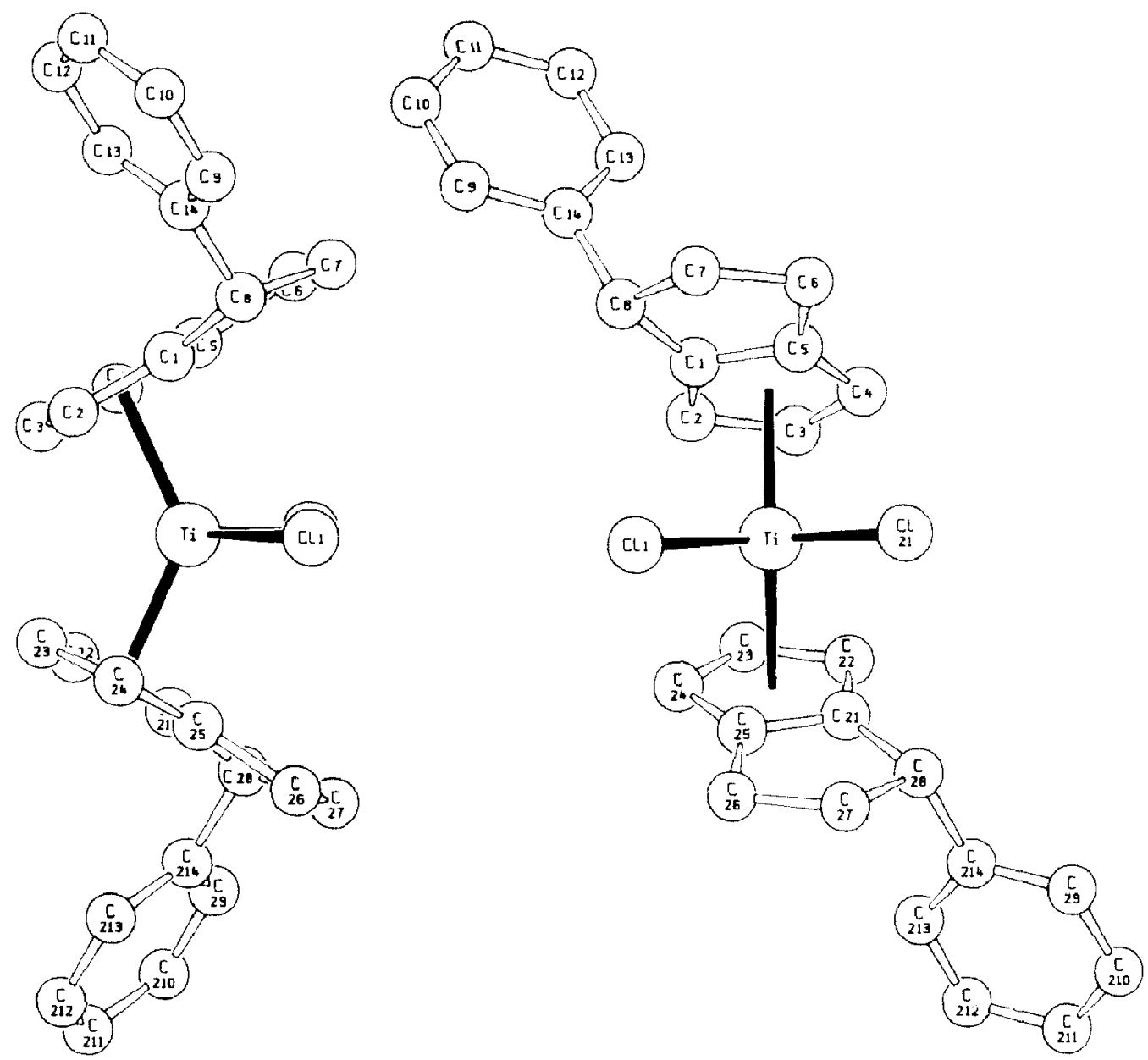

Fig. 1. Structure of racemic bis(4-exo-phenyl-4H-5,6-dihydropentalenide)titanium dichloride; projections perpendicular to centroid- $\mathrm{Ti}-$ centroid plane (left) and parallel to $\mathrm{Cl}-\mathrm{Ti}-\mathrm{Cl}$ bisector axis (right).

\section{Results and Discussion}

Reaction of 3-phenyl-1,2-dihydropentalene [7] with $\mathrm{Mg}$ metal and $\mathrm{CCl}_{4}$ in $\mathrm{THF}$ produces a cream-coloured organomagnesium reagent (ca. $30 \%$ theoretical yield). This compound reacts with one equivalent of $\mathrm{TiCl}_{3} \cdot 3 \mathrm{THF}$ in $\mathrm{THF}\left(30 \mathrm{~h}\right.$ at $\left.60^{\circ} \mathrm{C}\right)$ to yield, after oxidation with $\mathrm{HCl} / \mathrm{O}_{2}$ and extraction with $\mathrm{CH}_{2} \mathrm{Cl}_{2}$, a reddish product mixture. Flash chromatography on silica gel with toluene $/ \mathrm{CH}_{2} \mathrm{Cl}_{2}(2 / 1)$ yields a single red fraction, which upon evaporation gives a red crystalline solid in ca. $6 \%$ theoretical yield.

A crystal structural study of this material (Fig. 1) reveals the absence of the expected interannular bridge. The axially symmetric complex molecule instead contains two identical unconnected ring ligand moieties, which are derived from 3-phenyl-1,2-dihydropentalene by uptake of a hydride unit at the phenyl-substituted $\mathrm{C}$ atom*. The formation of analogous products of hydrogen uptake (rather than

\footnotetext{
* This benzylic hydrogen atom gives rise to a doublet of doublets at $4.52 \mathrm{ppm}$ in the ${ }^{1} \mathrm{H}$ NMR spectrum
} of this complex (Tab. 2). 
$\mathrm{C}-\mathrm{C}$ coupled products) has been noted previously in pentafulvene reductions under more strongly reducing conditions $[8,9]$, but not in $\mathrm{Mg} / \mathrm{CCl}_{4}$-induced reactions. Apparently, a phenyl substituent at the exocyclic double bond renders the generation of a product of hydrogen-uptake (possibly via a dianionic intermediate [9]) competitive with the desired coupling of two radical anions.

The bicyclic anionic ligand formed from phenyl-dihydropentalene by hydride uptake contains an asymmetric benzylic $\mathrm{C}$ atom; it coordinates to the Ti-centre preferentially in an exo fashion for obvious steric reasons. Why a racemic complex is formed by coordination of two equally-handed ligand moieties (rather than a meso combination of two ligands of oppositely configuration) is not clear, however. If present in the initial product mixture, such a meso species might have been lost in the subsequent purification procedure. In contrast, the product of a $\mathrm{Mg} / \mathrm{CCl}_{4}$-induced reduction of guaiazulene, reacts with $\mathrm{TiCl}_{3} \cdot 3 \mathrm{THF}$ to produce, after work-up and chromatography as described above, a deep violet crystalline complex, the crystallographically-determined structure of which (Fig. 2) reveals the presence of a $\mathrm{C}-\mathrm{C}$-coupled ansa-metallocene, 8,8' -biguaiazulenide titanium dichloride.

Of the two possible points of interannular $\mathrm{C}-\mathrm{C}$ coupling the unsubstituted 8-position is evidently preferred over the methyl-substituted 4-position, indicating again a tendency to avoid substituents at the coupling site. The two mutually connected 7-membered rings, each of which contains two double bonds, appear to be somewhat twisted; the interannular $\mathrm{C}-\mathrm{C}$ bond is slightly lengthened to $156-157$ pm (Tab. 3) ${ }^{*}$. The ${ }^{1} \mathrm{H}$ NMR signal of the $\mathrm{H}$-atoms attached to these bridging $\mathrm{C}$ atoms appears as a singlet at $4.61 \mathrm{ppm}$ (Tab. 4).

Except for the disordered conformations of the peripheral isopropyl groups, the complexes again show the axial symmetry expected for a racemic ansa-metallocene. The exclusive formation of a racemic rather than of a meso complex is noteworthy; it remains to be clarified whether this stereoselectivity originates from the initial $\mathrm{Mg} / \mathrm{CCl}_{4}$ coupling reaction or from elimination of any meso product in a subsequent reaction or during purification.

\section{Experimental}

3-Phenyl-1,2-dihydropentalene was prepared as described by Hafner and Kaiser [7]; the yield for the synthesis of 6-(2-dimethylaminovinyl)fulvene from cyclopentadiene and 3-dimethylaminoacrolein was improved from $30 \%$ [7] to $53 \%$ by utilizing the pyrrolidine-induced condensation procedure described by Stone and Little [10]. Guaiazulene was purchased from Aldrich and used without further purification. The procedures described below were, unless otherwise stated, carried out in well-dried glass vessels with anhydrous solvents under $\mathrm{N}_{2}$ or Ar.

1. Reaction of 3-phenyl-1,2-dihydropentalene with $\mathrm{Mg} / \mathrm{CCl}_{4}$. To $3.4 \mathrm{~g}$ of $\mathrm{Mg}$ turnings a solution of $21.7 \mathrm{~g}(0.12 \mathrm{~mol})$ 3-phenyl-1,2-dihydropentalene and $2 \mathrm{ml}$ of $\mathrm{CCl}_{4}$ in $45 \mathrm{ml}$ of THF was added slowly in small portions. After a brief induction period the mixture begins to boil; and it was kept at the reflux temperature by external heating during addition of the remaining THF solution. The solution was subsequently stirred at room temperature overnight then $25 \mathrm{ml}$ of anhydrous diethyl

\footnotetext{
* Similarly lengthened interannular $\mathrm{C}-\mathrm{C}$ bonds have been reported for both biazulenide [4] and bipentalenide iron(II) [5].
} 

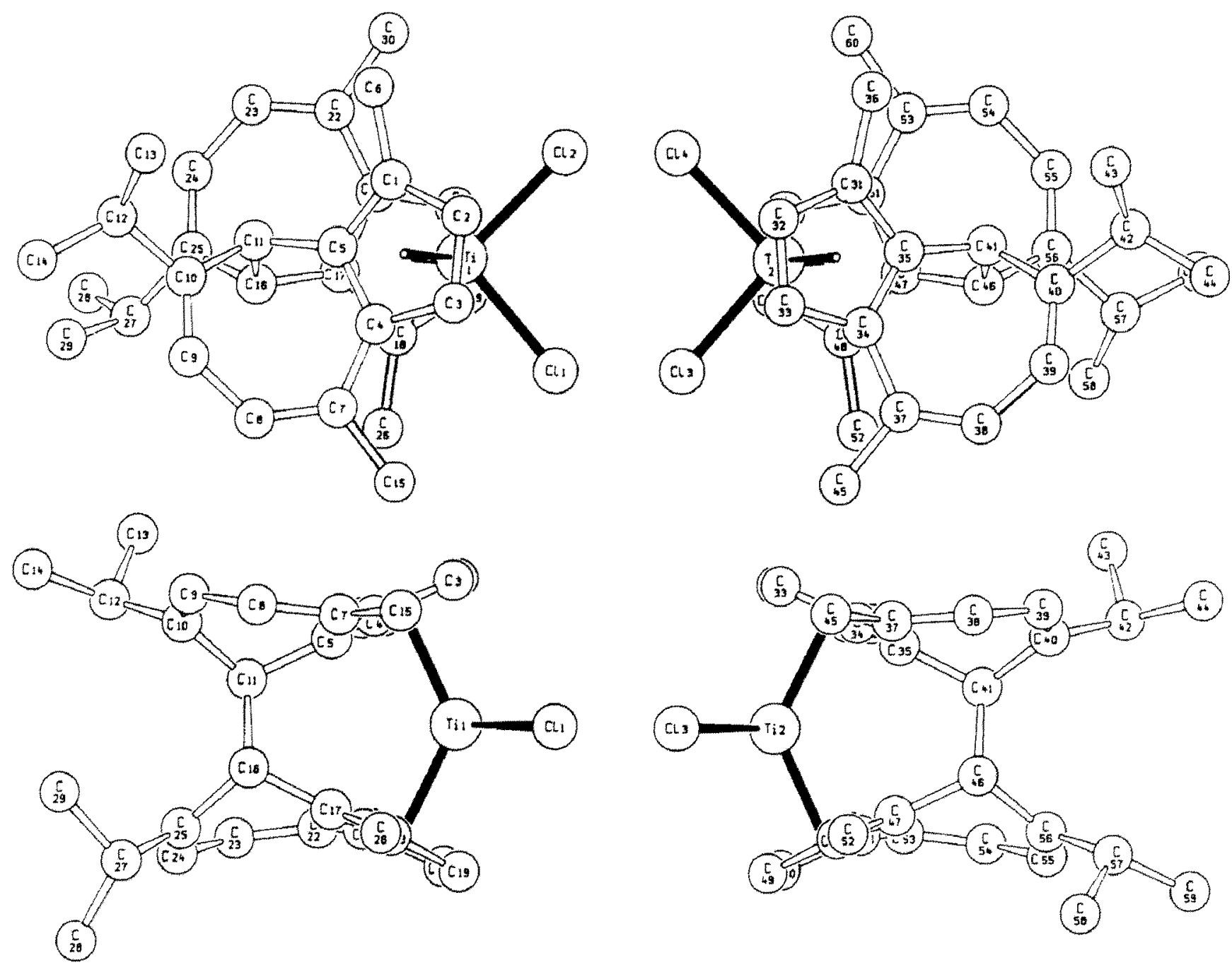

Fig. 2. Structures of two crystallographically independent molecules of racemic $8,8^{\prime}$-biguaiazulenide titanium dichloride, with $R$-configuration (left) and $S$-configuration (right); projections onto $\mathrm{TiCl}_{2}$ plane (top) and onto centroid-Ti-centroid plane (bottom). 
Table 1

Selected bond lengths (in pm) and bond and dihedral angles $\left({ }^{\circ}\right)$ for bis(4-exo-phenyl-4 $\mathrm{H}$-5,6-dihydropentalenide)titanium dichloride ( $C R=$ centroid, $P L=$ mean plane of $\eta^{5}-C_{5}$ ring)

\begin{tabular}{lllc}
\hline $\operatorname{Ti}(1)-C l(1)$ & $235.0(2)$ & $\operatorname{Ti}(1)-\mathrm{CR}(1)$ & 209.5 \\
$\operatorname{Ti}(1)-C(1)$ & $247.3(3)$ & & \\
$\operatorname{Ti}(1)-C(2)$ & $236.8(4)$ & $\mathrm{Cl}(1)-\operatorname{Ti}(1)-\mathrm{Cl}\left(1^{\prime}\right)$ & $95.9(1)$ \\
$\operatorname{Ti}(1)-\mathrm{C}(3)$ & $235.6(4)$ & $\mathrm{CR}(1)-\operatorname{Ti}(1)-\mathrm{CR}\left(1^{\prime}\right)$ & 131.0 \\
$\operatorname{Ti}(1)-C(4)$ & $240.0(4)$ & PL(1)-PL(1') & 57.2 \\
$\operatorname{Ti}(1)-C(5)$ & $248.0(3)$ & & \\
\hline
\end{tabular}

ether were added and the cream-coloured suspension was decanted from remaining $\mathrm{Mg}$ turnings and filtered. The pink solid residue was washed four times with $15 \mathrm{ml}$ portions of anhydrous diethyl ether and dried in vacuo to yield $14.3 \mathrm{~g} \mathrm{(31 \% )}$ of the air- and moisture-sensitive Grignard product.

2. Reaction of guaiazulene with $\mathrm{Mg} / \mathrm{CCl}_{4}$. An essentially analogous procedure as that described in 1 was carried out with $15 \mathrm{~g}(76 \mathrm{mmol})$ guaiazulene and $1.25 \mathrm{ml}$ $\mathrm{CCl}_{4}$ in $50 \mathrm{ml}$ anhydrous THF being added to $1.84 \mathrm{~g} \mathrm{(76} \mathrm{mmol)} \mathrm{of} \mathrm{Mg}$ turnings to yield, after work-up as described above, $9.5 \mathrm{~g}(31 \%)$ of the bluish-white organomagnesium reagent.

3. Synthesis of bis(4-phenyl-4,5,6-trihydropentalenide)titanium dichloride. A THF solution of $10.6 \mathrm{~g}(13.8 \mathrm{mmol})$ of the organomagnesium reagent derived from 3-phenyl-1,2-dihydropentalene and $5.1 \mathrm{~g}(13.8 \mathrm{mmol})$ of $\mathrm{TiCl}_{3} \cdot 3 \mathrm{THF}$ was refluxed for $30 \mathrm{~h}$ then cooled to $-40^{\circ} \mathrm{C}$, treated with $3 \mathrm{ml} 5 \mathrm{M} \mathrm{HCl}$, stirred at room temperature with exposure to air for $8 \mathrm{~h}$, then evaporated to dryness. Without protection from the air the solid residue was taken up in $40 \mathrm{ml} \mathrm{CH}_{2} \mathrm{Cl}_{2}$ and $15 \mathrm{ml} 5$ $M \mathrm{HCl}$. The organic layer was separated, dried over $\mathrm{MgSO}_{4}$, and evaporated to dryness. Flash chromatography of the residue on a silica gel column with toluene/ $\mathrm{CH}_{2} \mathrm{Cl}_{2}(2 / 1)$ yielded first a yellow fraction (discarded) and then a red fraction, which was dried over $\mathrm{MgSO}_{4}$ and evaporated in vacuo to yield $0.40 \mathrm{~g}(6 \%)$ of $R, S$-bis(4-phenyl-4H-5,6-dihydro-pentalenide)titanium dichloride. The ${ }^{1} \mathbf{H}$ NMR data are shown in Table 2 and results of the X-ray structure determination in Table 1 ; the mass spectrum showed ions at $m / e 446\left(M^{+}-\mathrm{Cl}\right)$ and $411\left(M^{+}-2 \mathrm{Cl}\right)$ with the expected isotope patterns. Analysis. Found: C, 69.39; H, 5.45. $\mathrm{C}_{28} \mathrm{H}_{26} \mathrm{TiCl}_{2}$ calcd.: C, 69.85; H, 5.43\%.

Table 2

${ }^{1} \mathrm{H}$ NMR spectrum of bis(4-phenyl-5,6-dihydropentalenide)titanium dichloride in $\mathrm{CDCl}_{3}$ solution at $25^{\circ} \mathrm{C}, \delta$ in ppm at $250 \mathrm{MHz}$ ( $\mathrm{m}=$ multiplet, $\mathrm{pt}=$ psdeudotriplet with $J(\mathrm{H}, \mathrm{H}) \approx 3 \mathrm{~Hz}, \mathrm{dd}=$ doublet of doublets $\mathrm{J}(\mathrm{H}, \mathrm{H}) \approx 3.6$ and $3.0 \mathrm{~Hz})$

\begin{tabular}{llllll}
\hline 7.07 & & & 4.52 & $(\mathrm{dd} 2 \mathrm{H})$ & $\mathrm{C}_{\left(\mathrm{C}_{6} \mathrm{H}_{5}\right) \mathrm{H}-\mathrm{CH}_{2}-\mathrm{CH}_{2}}$ \\
to & $(\mathrm{m}, 10 \mathrm{H})$ & $\mathrm{C}_{6} H_{5}$ & 2.76 & \\
7.31 & & & to & $(\mathrm{m}, 6 \mathrm{H})$ & $\mathrm{C}\left(\mathrm{C}_{6} \mathrm{H}_{5}\right) \mathrm{H}-\mathrm{CH}_{2}-\mathrm{CH}_{2}$ \\
& & 3.05 & & \\
6.57 & $(\mathrm{pt}, 2 \mathrm{H})$ & $\eta^{5}-\mathrm{C}_{5} \mathrm{H}_{3}$ & 2.27 & \\
5.90 & $(\mathrm{pt}, 2 \mathrm{H})$ & $\eta^{5}-\mathrm{C}_{5} \mathrm{H}_{3}$ & to & $(\mathrm{m}, 2 \mathrm{H})$ & $\mathrm{C}\left(\mathrm{C}_{6} \mathrm{H}_{5}\right) \mathrm{H}-\mathrm{CH}_{2}-\mathrm{CH}_{2}$ \\
5.77 & $(\mathrm{pt}, 2 \mathrm{H})$ & $\eta^{5}-\mathrm{C}_{5} \mathrm{H}_{3}$ & 2.32 & \\
\hline
\end{tabular}


Table 3

Selected bond lengths (in pm) and bond and dihedral angles (in degree) for $8,8^{\prime}$-biguaiazulenidetitanium dichloride $\left(C R=\right.$ centroid, $\mathrm{PL}=$ mean plane of $\eta^{5}-C_{5}$ ring $)$.

\begin{tabular}{|c|c|c|c|}
\hline $\mathrm{Ti}(1)-\mathrm{Cl}(1)$ & $235.1(1)$ & $\mathrm{Ti}(2)-\mathrm{Cl}(3)$ & $235.3(1)$ \\
\hline $\mathrm{Ti}(1)-\mathrm{Cl}(2)$ & $235.8(1)$ & $\operatorname{Ti}(2)-\mathrm{Cl}(4)$ & $235.8(1)$ \\
\hline $\operatorname{Ti}(1)-\mathrm{C}(1)$ & $239.9(2)$ & $\mathrm{Ti}(2)-\mathrm{C}(31)$ & $240.2(2)$ \\
\hline $\operatorname{Ti}(1)-C(2)$ & $240.4(2)$ & $\mathrm{Ti}(2)-\mathrm{C}(32)$ & $239.1(2)$ \\
\hline $\operatorname{Ti}(1)-C(3)$ & $239.1(3)$ & $\operatorname{Ti}(2)-C(33)$ & $240.1(3)$ \\
\hline $\operatorname{Ti}(1)-C(4)$ & $242.3(3)$ & $\operatorname{Ti}(2)-\mathrm{C}(34)$ & $242.3(3)$ \\
\hline $\mathrm{Ti}(1)-\mathrm{C}(5)$ & $240.7(3)$ & $\mathrm{Ti}(2)-\mathrm{C}(35)$ & $241.9(3)$ \\
\hline $\operatorname{Ti}(1)-C(17)$ & $241.7(3)$ & $\operatorname{Ti}(2)-C(47)$ & $241.7(3)$ \\
\hline $\mathrm{Ti}(1)-\mathrm{C}(18)$ & $239.8(3)$ & $\operatorname{Ti}(2)-C(48)$ & $239.7(3)$ \\
\hline $\mathrm{Ti}(1)-\mathrm{C}(19)$ & $239.8(2)$ & $\operatorname{Ti}(2)-C(49)$ & $240.5(3)$ \\
\hline $\operatorname{Ti}(1)-C(20)$ & $239.6(3)$ & $\mathrm{Ti}(2)-\mathrm{C}(50)$ & $240.8(3)$ \\
\hline $\operatorname{Ti}(1)-C(21)$ & $242.7(3)$ & $\mathrm{Ti}(2)-\mathrm{C}(51)$ & $242.9(3)$ \\
\hline $\operatorname{Ti}(1)-C R(1)$ & 208.1 & $\operatorname{Ti}(2)-\mathrm{CR}(3)$ & 208.4 \\
\hline $\operatorname{Ti}(1)-C R(2)$ & 208.5 & $\operatorname{Ti}(2)-\mathrm{CR}(4)$ & 209.0 \\
\hline $\mathrm{Cl}(1)-\mathrm{Ti}(1)-\mathrm{Cl}(2)$ & $95.0(1)$ & $\mathrm{Cl}(3)-\mathrm{Ti}(2)-\mathrm{Cl}(4)$ & $95.1(1)$ \\
\hline $\mathrm{CR}(1)-\mathrm{Ti}(1)-\mathrm{CR}(2)$ & 128.5 & $\mathrm{CR}(3)-\mathrm{Ti}(2)-\mathrm{CR}(4)$ & 128.9 \\
\hline $\operatorname{PL}(1)-P L(2)$ & 50.8 & $\mathrm{PL}(3)-\mathrm{PL}(4)$ & 50.3 \\
\hline
\end{tabular}

4. Synthesis of $8,8^{\prime}$-biguaiazulenidetitanium dichloride. A similar procedure of 9.5 $\mathrm{g}(11.8 \mathrm{mmol})$ of the organomagnesium reagent derived from guaiazulene and $4.4 \mathrm{~g}$ $\left(11.8 \mathrm{mmol}\right.$ ) of $\mathrm{TiCl}_{3} \cdot 3 \mathrm{THF}$ gave, after work-up and flash chromatography as described above, two blue fractions. The first contained mainly hydrolysed ligand, while the second yielded, upon evaporation, $0.60 \mathrm{~g}(9.9 \%)$ of violet-black crystalline $8,8^{\prime}$-biguaiazulenide titanium dichloride. The ${ }^{1} \mathrm{H}$ NMR data are shown in Table 4 and the results of the $\mathrm{X}$-ray structure determination in Table 3. The mass spectrum showed the parent ion at $m / e 514$ and a fragment ion at $m / e 476\left(\mathrm{M}^{+}-\mathrm{Cl}\right)$ with the expected isotope pattern.

5. Crystal structure determinations. Space groups, cell parameters and X-ray diffraction intensities of suitable crystals of bis(4-phenyl-4 $\mathrm{H}$-5,6-dihydropentalenide)titanium dichloride and of 8,8'-biguaiazulenidetitanium dichloride were determined on a Syntex P3 four circle diffractometer ( $\mathrm{Mo}-K_{\alpha}, \lambda 71.069 \mathrm{ppm}$, graphite

Table 4

${ }^{1} \mathrm{H}$ NMR spectrum of $8,8^{\prime}$-biguaiazulenidetitanium dichloride in $\mathrm{CDCl}_{3}$ solution at $25^{\circ} \mathrm{C}, \delta$ in ppm at $250 \mathrm{MHz}(\mathrm{s}=$ singlet, $\mathrm{d}=$ doublet, $\mathrm{sp}=$ septet $)$

\begin{tabular}{llllll}
\hline 7.05 & $(\mathrm{~d}, 2 \mathrm{H})^{a}$ & $\eta^{5}-\mathrm{C}_{5} \mathrm{H}_{2}$ & 2.32 & $(\mathrm{sp}, 2 \mathrm{H})^{b}$ & $7-\mathrm{CH}\left(\mathrm{CH}_{3}\right)_{2}$ \\
6.60 & $(\mathrm{~d}, 2 \mathrm{H})^{a}$ & $\eta^{5}-\mathrm{C}_{5} \mathrm{H}_{2}$ & & & \\
& & & 2.26 & $(\mathrm{~s}, 6 \mathrm{H})$ & $1,4-\mathrm{CH}_{3}$ \\
6.31 & $(\mathrm{~d}, 2 \mathrm{H})^{b}$ & $5,6-\mathrm{C}_{2} \mathrm{H}_{2}$ & 2.21 & $(\mathrm{~s}, 6 \mathrm{H})$ & $1,4-\mathrm{CH}_{3}$ \\
5.92 & $(\mathrm{~d}, 2 \mathrm{H})^{b}$ & $5,6-\mathrm{C}_{2} \mathrm{H}_{2}$ & & & \\
& & & & & \\
4.61 & $(\mathrm{~s}, 2 \mathrm{H})$ & $8-\mathrm{CH}$ & 0.93 & $(\mathrm{~d}, 6 \mathrm{H})^{b}$ & $7-\mathrm{CH}\left(\mathrm{CH}_{3}\right)_{2}$ \\
& & &
\end{tabular}

${ }^{a} J(\mathrm{H}, \mathrm{H}) \approx 2.5 \mathrm{~Hz} .{ }^{b} J(\mathrm{H}, \mathrm{H}) \approx 7.0 \mathrm{~Hz}$. 
Table 5

Atomic coordinates $\left(\times 10^{4}\right)$ and isotropic thermal parameters (in $\AA^{2} \times 10^{3}$ ) for bis(4-exo-phenyl-4H-5,6dihydropentalenide)titanium dichloride

\begin{tabular}{lllll}
\hline & $x$ & $y$ & $z$ & $U$ \\
\hline $\mathrm{Ti}(1)$ & 5000 & $5936(1)$ & 2500 & $26(1)^{a}$ \\
$\mathrm{C}(1)$ & $5068(1)$ & $8302(1)$ & $1102(1)$ & $36(1)^{a}$ \\
$\mathrm{C}(1)$ & $5830(1)$ & $5593(5)$ & $2337(3)$ & $30(1)^{a}$ \\
$\mathrm{C}(2)$ & $5589(1)$ & $3736(5)$ & $2106(3)$ & $34(1)^{a}$ \\
$\mathrm{C}(3)$ & $5458(1)$ & $3111(5)$ & $3132(3)$ & $41(1)^{a}$ \\
$\mathrm{C}(4)$ & $5585(1)$ & $4596(5)$ & $3946(3)$ & $41(1)^{a}$ \\
$\mathrm{C}(5)$ & $5824(1)$ & $6118(5)$ & $3440(3)$ & $35(1)^{a}$ \\
$\mathrm{C}(6)$ & $6107(1)$ & $7988(6)$ & $3775(3)$ & $48(1)^{a}$ \\
$\mathrm{C}(7)$ & $6232(1)$ & $8720(5)$ & $2657(3)$ & $45(1)^{a}$ \\
$\mathrm{C}(8)$ & $6136(1)$ & $6974(5)$ & $1784(3)$ & $36(1)^{a}$ \\
$\mathrm{C}(9)$ & $6764(1)$ & $6401(3)$ & $606(2)$ & $47(1)$ \\
$\mathrm{C}(10)$ & 7163 & 5432 & 395 & $52(1)$ \\
$\mathrm{C}(11)$ & 7375 & 3954 & 1125 & $53(1)$ \\
$\mathrm{C}(12)$ & 7186 & 3446 & 2067 & $54(1)$ \\
$\mathrm{C}(13)$ & 6786 & 4416 & 2278 & $47(1)$ \\
$\mathrm{C}(14)$ & 6575 & 5893 & 1548 & $34(1)$ \\
\hline
\end{tabular}

a Equivalent isotropic $U$ defined as one third of the trace of the orthogonalised $U_{i j}$ tensor

monochromator, $\omega$-scan with $\Delta \omega 1^{\circ}\left(2.0<\dot{\omega}<10.0^{\circ} \cdot \min ^{-1} ; 3.0<2 \theta<53^{\circ}\right.$ and $2.2<\dot{\omega}<29.3^{\circ} \cdot \min ^{-1}, 4.0<2 \theta<50^{\circ}$, respectively).

The crystals of bis(4-phenyl-4H-5,6-dihydropentalenide)titanium dichloride, examined at $236 \mathrm{~K}$, were monoclinic, space group $C 2 / c$ with $a 2917(2), b 665.1(3), c$ 1216.2(8) pm, $\beta$ 99.94(5) grad, $V 2424 \times 10^{6} \mathrm{pm}^{3}, 4$ crystallographically equivalent molecules per unit cell, $d_{\text {calcd. }} 1.27 \mathrm{~g} / \mathrm{cm}^{3}$. For resolution and refinement of the structure, 1829 independent reflections with $I>1.5 \sigma(I)$ were used, without absorption corrections. The structure was solved by direct methods (SHELXTL). The Ti atom is situated on a crystallographic $C_{2}$ axis. Refinement with a partially anisotropic model, with the phenyl ring fixed in its hexagonal geometry and with all $\mathrm{H}$ atoms in calculated positions converged at $R_{1}=0.053$ and $R_{2}=0.044$. The resulting structural parameters are listed in Tab. 5.

The crystals of $8,8^{\prime}$-biguaiazulenidetitanium dichloride were triclinic, space group $P \overline{1}$ with $a$ 1295.9(2), $b$ 1318.3(2), c 1571.8(2) pm, $\alpha$ 95.02(1), $\beta$ 97.70(1), $\gamma$ $103.11(1)^{\circ} ; V 2572 \times 10^{6} \mathrm{pm}^{3}$, two pairs of crystallographically equivalent molecules per unit cell; $d_{\text {calcd. }} 1.33 \mathrm{~g} / \mathrm{cm}^{3}$. For resolution and refinement of the structure, 7144 independent reflections with $I>1.5 \sigma(I)$ were used, without absorption corrections. The structure was solved by direct methods (SHELXTL). Refinement with a partially anisotropic model (omitting reflections $3-23,-634,-531$ and 3 -2 2) converged at $R_{1}=0.042$ and $R_{2}=0.042$ ( $\mathrm{H}$ atoms in calculated positions) * The resulting structural parameters are represented in Tab. 6.

Additional structural data for these two compounds are available on request from Fachinformationszentrum Energie Physik Mathematik, D-7514 Eggenstein- 
160

Table 6

Atomic coordinates $\left(\times 10^{4}\right)$ and isotropic thermal parameters (in $\AA^{2} \times 10^{3}$ ) for $8,8^{\prime}$-biguaiazulenide titanium dichloride

\begin{tabular}{|c|c|c|c|c|}
\hline & $x$ & $y$ & $z$ & $U^{a}$ \\
\hline $\mathrm{Ti}(1)$ & $3354(1)$ & $3059(1)$ & $2798(1)$ & $21(1)$ \\
\hline $\mathrm{Cl}(\mathbf{1})$ & $1838(1)$ & $3362(1)$ & $1953(1)$ & $39(1)$ \\
\hline $\mathrm{Cl}(2)$ & $2848(1)$ & $3526(1)$ & $4136(1)$ & $42(1)$ \\
\hline$C(1)$ & $5129(2)$ & $4096(2)$ & $3356(2)$ & $24(1)$ \\
\hline$C(2)$ & $4471(2)$ & $4804(2)$ & $3197(2)$ & $28(1)$ \\
\hline$C(3)$ & $4101(2)$ & $4699(2)$ & $2317(2)$ & $28(1)$ \\
\hline$C(4)$ & $4550(2)$ & $3945(2)$ & $1888(2)$ & $25(1)$ \\
\hline$C(5)$ & $5181(2)$ & $3560(2)$ & $2543(2)$ & $21(1)$ \\
\hline$C(6)$ & $5754(3)$ & $4048(2)$ & $4222(5)$ & $32(1)$ \\
\hline$C(7)$ & $4516(2)$ & $3751(2)$ & $955(2)$ & $29(1)$ \\
\hline$C(8)$ & $5341(3)$ & $3512(2)$ & $612(2)$ & $33(1)$ \\
\hline$C(9)$ & $6372(3)$ & $3402(2)$ & $1033(2)$ & $32(1)$ \\
\hline$C(10)$ & $6631(2)$ & $3104(2)$ & $1817(2)$ & $25(1)$ \\
\hline$C(11)$ & $5785(2)$ & $2733(2)$ & $2377(2)$ & $20(1)$ \\
\hline$C(12)$ & $7769(2)$ & $3107(2)$ & $2205(2)$ & $32(1)$ \\
\hline$C(13)$ & $8215(3)$ & $4042(3)$ & $2911(2)$ & $41(1)$ \\
\hline$C(14)$ & $8538(3)$ & $3108(3)$ & $1550(3)$ & $47(1)$ \\
\hline$C(15)$ & $3593(3)$ & 3994(3) & $381(2)$ & $45(1)$ \\
\hline$C(16)$ & $4988(2)$ & $1670(2)$ & $1961(2)$ & $20(1)$ \\
\hline$C(17)$ & $3963(2)$ & $1535(2)$ & $2319(2)$ & $19(1)$ \\
\hline$C(18)$ & $2917(2)$ & $1434(2)$ & $1861(2)$ & 23(1) \\
\hline$C(19)$ & $2203(2)$ & $1326(2)$ & $2475(2)$ & $28(1)$ \\
\hline$C(20)$ & $2785(2)$ & $1383(2)$ & $3291(2)$ & $26(1)$ \\
\hline$C(21)$ & $3886(2)$ & $1500(2)$ & $3217(2)$ & $22(1)$ \\
\hline$C(22)$ & $4724(2)$ & $1387(2)$ & $3904(2)$ & $27(1)$ \\
\hline$C(23)$ & $5505(2)$ & $922(2)$ & $3723(2)$ & $29(1)$ \\
\hline$C(24)$ & $5661(2)$ & $443(2)$ & $2897(2)$ & $29(1)$ \\
\hline$C(25)$ & $5418(2)$ & $702(2)$ & $2101(2)$ & $24(1)$ \\
\hline$C(26)$ & $2597(2)$ & $1353(2)$ & $903(2)$ & $31(1)$ \\
\hline$C(27)$ & $5504(3)$ & $26(2)$ & $1290(2)$ & $33(1)$ \\
\hline$C(28)$ & $5540(4)$ & $-1102(3)$ & $1411(3)$ & $56(2)$ \\
\hline$C(29)$ & $6451(3)$ & $548(3)$ & $875(2)$ & $43(1)$ \\
\hline$C(30)$ & $4570(3)$ & $1653(3)$ & $4824(2)$ & $37(1)$ \\
\hline $\operatorname{Ti}(2)$ & 1741(1) & $7155(1)$ & $2271(1)$ & $22(1)$ \\
\hline $\mathrm{Cl}(3)$ & $3218(1)$ & 6856(1) & $3172(1)$ & $39(1)$ \\
\hline $\mathrm{Cl}(4)$ & 2301(1) & $6646(1)$ & $967(1)$ & 41(1) \\
\hline$C(31)$ & $-29(2)$ & $6117(2)$ & $1682(2)$ & $24(1)$ \\
\hline$C(32)$ & $632(2)$ & $5417(2)$ & $1880(2)$ & $27(1)$ \\
\hline$C(33)$ & $956(2)$ & $5527(2)$ & $2768(2)$ & $28(1)$ \\
\hline$C(34)$ & $493(2)$ & $6299(2)$ & $3156(2)$ & $25(1)$ \\
\hline$C(35)$ & $-115(2)$ & $6667(2)$ & $2480(2)$ & $22(1)$ \\
\hline$C(36)$ & $-627(3)$ & $6138(2)$ & $803(2)$ & $31(1)$ \\
\hline$C(37)$ & $477(3)$ & $6499(2)$ & $4090(2)$ & $32(1)$ \\
\hline$C(38)$ & $-348(3)$ & $6775(2)$ & $4397(2)$ & $35(1)$ \\
\hline$C(39)$ & $-1350(3)$ & $6932(2)$ & $3953(2)$ & $31(1)$ \\
\hline$C(40)$ & $-1580(2)$ & $7206(2)$ & $3156(2)$ & $26(1)$ \\
\hline $\mathrm{C}(41)$ & $-720(2)$ & $7502(2)$ & $2601(2)$ & $20(1)$ \\
\hline$C(42)$ & $-2694(2)$ & $7275(3)$ & $2775(2)$ & $33(1)$ \\
\hline$C(43)$ & $-3221(3)$ & $6314(3)$ & $2113(2)$ & $49(1)$ \\
\hline$C(44)$ & $-3432(3)$ & $7411(3)$ & $3432(3)$ & $54(2)$ \\
\hline$C(45)$ & $1367(3)$ & $6238(3)$ & $4689(2)$ & $48(1)$ \\
\hline$C(46)$ & $58(2)$ & $8581(2)$ & $2977(2)$ & $20(1)$ \\
\hline
\end{tabular}


Table 6 (continued)

\begin{tabular}{lrrll}
\hline & $x$ & $y$ & $z$ & $U^{a}$ \\
\hline $\mathrm{C}(47)$ & $1121(2)$ & $8702(2)$ & $2665(2)$ & $20(1)$ \\
$\mathrm{C}(48)$ & $2133(2)$ & $8798(2)$ & $3176(2)$ & $25(1)$ \\
$\mathrm{C}(49)$ & $2902(2)$ & $8888(2)$ & $2610(2)$ & $29(1)$ \\
$\mathrm{C}(50)$ & $2388(2)$ & $8822(2)$ & $1771(2)$ & $30(1)$ \\
$\mathrm{C}(51)$ & $1271(2)$ & $8714(2)$ & $1784(2)$ & $24(1)$ \\
$\mathrm{C}(52)$ & $2379(3)$ & $8883(2)$ & $4144(2)$ & $34(1)$ \\
$\mathrm{C}(53)$ & $470(2)$ & $8797(2)$ & $1058(2)$ & $26(1)$ \\
$\mathrm{C}(54)$ & $-341(2)$ & $9248(2)$ & $1170(2)$ & $27(1)$ \\
$\mathrm{C}(55)$ & $-613(2)$ & $9716(2)$ & $1957(2)$ & $27(1)$ \\
$\mathrm{C}(56)$ & $-427(2)$ & $9493(2)$ & $2774(2)$ & $22(1)$ \\
$\mathrm{C}(57)$ & $-702(2)$ & $10106(2)$ & $3544(2)$ & $29(1)$ \\
$\mathrm{C}(58)$ & $297(3)$ & $10739(3)$ & $4136(2)$ & $40(1)$ \\
$\mathrm{C}(59)$ & $-1439(3)$ & $10829(3)$ & $3306(3)$ & $56(2)$ \\
$\mathrm{C}(60)$ & $678(3)$ & $8486(2)$ & $159(2)$ & $34(1)$ \\
\hline
\end{tabular}

${ }^{a}$ Equivalent isotropic $U$ defined as one third of the trace of the orthogonalised $U_{i j}$ tensor

Leopoldshafen 2 under quotation of deposit No. CSD 53830, the authors and the journal reference of this article.

\section{Acknowledgements}

Financial support for this work by Deutsche Forschungsgemeinschaft (grant $\mathrm{Br}$ 510), by Fonds der Chemischen Industrie and by funds of the University of Konstanz is gratefully acknowledged.

\section{References}

1 H. Wiesenfeldt, A. Reinmuth, E. Barsties, K. Evertz and H.H. Brintzinger, J. Organomet. Chem., 369 (1989) 359.

2 E.O. Fischer and J. Müller, J. Organomet. Chem., 1 (1964) 464.

3 T.J. Katz, N. Acton and J. McGinnis, J. Am. Chem. Soc., 94 (1972) 6205.

4 M.R. Churchill and J. Wormald, Chem. Comm., (1968) 1033; Inorg. Chem., 8 (1969) 716.

5 M.R. Churchill and K.K.G. Lin, Inorg. Chem., 12 (1973) 2274.

6 S. Gutmann, P. Burger, H.U. Hund, J. Hofmann and H.H. Brintzinger J. Organomet. Chem., 369 (1989) 343.

7 K. Hafner and R. Kaiser, Angew. Chem., 82 (1970) 877.

8 M.F. Sullivan and W.F. Little, J. Organomet. Chem., 8 (1967) 277.

9 H. Schwemlein and H.H. Brintzinger, J. Organomet. Chem., 254 (1983) 69.

10 K.J. Stone and R.D. Little, J. Org. Chem., 49 (1984) 1849. 